\title{
Post-Newtonian limit: second-order Jefimenko equations
}

\author{
David Pérez Carlos ${ }^{1, a}$, Augusto Espinoza ${ }^{2}$, Andrew Chubykalo ${ }^{2}$ \\ ${ }^{1}$ Unidad Académica de Física, Universidad Autónoma de Zacatecas, Campus II, Av. Preparatoria s/n, 98068 Zacatecas, Mexico \\ ${ }^{2}$ Unidad Académica de Ciencia y Tecnología de Luz y Materia, Universidad Autónoma de Zacatecas, Ciudad Universitaria Campus Siglo XX1, \\ 98160 Zacatecas, Mexico
}

Received: 28 October 2019 / Accepted: 10 July 2020 / Published online: 21 July 2020

(C) The Author(s) 2020

\begin{abstract}
The purpose of this paper is to get second-order gravitational equations, a correction made to Jefimenko's linear gravitational equations. These linear equations were first proposed by Oliver Heaviside in [1], making an analogy between the laws of electromagnetism and gravitation. To achieve our goal, we will use perturbation methods on Einstein field equations. It should be emphasized that the resulting system of equations can also be derived from Logunov's non-linear gravitational equations, but with different physical interpretation, for while in the former gravitation is considered as a deformation of space-time as we can see in [2-5], in the latter gravitation is considered as a physical tensor field in the Minkowski space-time (as in [6-8]). In Jefimenko's theory of gravitation, exposed in $[9,10]$, there are two kinds of gravitational fields, the ordinary gravitational field, due to the presence of masses, at rest, or in motion and other field called Heaviside field due to and acts only on moving masses. The Heaviside field is known in general relativity as Lense-Thirring effect or gravitomagnetism (The Heaviside field is the gravitational analogous of the magnetic field in the electromagnetic theory, its existence was proved employing the Gravity Probe B launched by NASA (See, for example, $[11,12])$. It is a type of gravitational induction), interpreted as a distortion of space-time due to the motion of mass distributions, (see, for example [13,14]). Here, we will present our second-order Jefimenko equations for gravitation and its solutions.
\end{abstract}

\section{Introduction}

In general relativity gravitational interaction is interpreted as deformation of space-time due to the presence and movement of masses. We can read in almost all books related to general relativity about the relationship existing between matter and space-time: "space-time tells matter how to move, matter

a e-mail: dperezcarlos @ gmail.com (corresponding author) tells to space-time how to curve" (see, [15]). This idea arose from the conclusion of Einstein that the field variable for the gravitational field must be the metric tensor of the Riemann space-time $g_{\mu \nu}$, and that this quantity is determined by the distribution and motion of matter, this is the link between matter and geometry. General relativity theory is often mentioned by various authors as one of the most important theories developed in the last century. In words of the Nobel prize R. Feynman: "Einstein's gravitational theory, which is said to be the greatest single achievement of theoretical physics, resulted in beautiful relations connecting gravitational phenomena with the geometry of space; this is an exciting idea." [16]. Although this relationship between matter and geometry of space-time has been widely spread between the most of scientist, this was not the central result that Einstein wanted to highlight, this idea never was accepted by Einstein, instead of this, was the unification of inertia and gravity. In fact, the idea of the geometrization of gravity began with the work of Weyl. For a detailed discussion, see the paper, Why Einstein did not believe that general relativity geometrizes gravity [17].

To obtain the second-order Jefimenko equations, we will use Post-Newtonian approximation on the Einstein field equations. The same system of non-linear equations can be obtained from other approaches such as the system of equations obtained by Logunov.

The Jefimenko equations for gravitation were first obtained by Heaviside in [1], making use of Gibbs vector analysis, supposing the existence of a second gravitational field arising as a kind of gravitational induction. Years before Einstein wrote about this possibility in his not-so-well-known work Gibt es eine gravitationwirkung die der elektromagnetischen induktionswirkung analog ist? [18]. But also can be obtained from the approach developed by Arbab in [19] making use of Hamilton's quaternions, from the linearised field equations as were obtained by González Segura in [20]. Jefimenko has obtained this set of equations by postulating an analogy 
between his retarded solutions for the electromagnetic field and solutions for the gravitational field.

We will compare the resulting system of equations with those quantities obtained by Jefimenko and we will establish their respective solutions as a limit. Also, we will see that the Jefimenko equations give us the Newtonian theory for the limit $v / c \rightarrow 0$, where $c$ is the speed of propagation of plane wave solutions of the gravitational field in the absence of sources ${ }^{1}$.

\section{Second-order Jefimenko equations}

Jefimenko equations for gravitation are given in $[9,10]$ by

$$
\begin{aligned}
\nabla \cdot \mathbf{g} & =-4 \pi G \rho, \\
\nabla \cdot \mathbf{k} & =0 \\
\nabla \times \mathbf{g} & =-\frac{\partial \mathbf{k}}{\partial t}, \\
\nabla \times \mathbf{k} & =-\frac{4 \pi G}{c^{2}} \mathbf{J}+\frac{1}{c^{2}} \frac{\partial \mathbf{g}}{\partial t},
\end{aligned}
$$

where $\mathbf{g}$ is the ordinary gravitational field due to the presence of masses and that acts on masses, whether they are moving or not, $\mathbf{k}$ is the Heaviside field (the field that is generated by the movement of masses and acts only on moving masses), $\rho$ is the mass density, $\mathbf{J}=\rho \mathbf{v}$ is the mass current density with $\mathbf{v}$ the velocity of the mass, $G$ is the universal constant of gravitation.

The analogy is not perfect, owing to that in electromagnetic theory there are two kinds of electric charges, in Jefimenko's theory of gravitation, there is only one kind of mass. Whereas in the first the magnetic field is right-handed, in gravitational theory is left-handed. In the former, there is a flux of electrons inside a conductor, while in the gravitation, the mass current is the movement of the matter as a whole.

We will derive non-linear expressions for the Jefimenko equations from the Einstein field equations:

$G_{\mu \nu}=8 \pi G T_{\mu \nu}$

where $G_{\mu \nu}=R_{\mu \nu}-\frac{1}{2} g_{\mu \nu} R$ is the Einstein tensor which determines the geometry of the space-time, due to the presence of the source, $G$ is the gravitational constant, $T_{\mu \nu}$ is the energy-momentum tensor of matter. These equations can be written as

$R_{\mu \nu}=8 \pi G\left(T_{\mu \nu}-\frac{1}{2} g_{\mu \nu} T\right)$,

where $T=T_{\mu}^{\mu}$ is the trace of the tensor $T_{\mu \nu}, R_{\mu \nu}$ is the Ricci tensor obtained from the contraction of the Riemann

\footnotetext{
1 This speed is considered to be equal to the speed of propagation of light. There are indirect measurements in [21].
}

tensor $R_{\mu \sigma v}^{\rho}$ for $\rho=\sigma$, that is,

$R_{\mu \nu}=R_{\mu \rho \nu}^{\rho}=\Gamma_{\mu \nu . \rho}^{\rho}-\Gamma_{\mu \sigma, v}^{\rho}+\Gamma_{\sigma \lambda}^{\rho} \Gamma_{\mu \nu}^{\lambda}-\Gamma_{\lambda \nu}^{\rho} \Gamma_{\mu \sigma}^{\lambda}$,

and $\Gamma_{\mu \nu}^{\rho}$ are the Christoffel symbols, given in terms of the metric tensor of the Riemann space-time $g_{\mu \nu}$ as

$\Gamma_{\mu \nu}^{\rho}=\frac{1}{2} g^{\rho \epsilon}\left(g_{\epsilon \mu, \nu}+g_{\epsilon \nu, \mu}-g_{\mu \nu, \epsilon}\right)$.

We will use the De Donder conditions

$\partial_{\mu} g^{\mu \nu}=0$

which were first introduced by Fock in [22] and were considered as a privileged system of reference. He introduced these conditions when he was considering problems of the island type (also called isolated systems). Until there are not global Cartesian coordinates in Riemann space-time, the harmonic gauge conditions are valid on patches.

To solve Eqs. (6) and (9), it is necessary to construct the Riemannian manifold, which means to find the metric tensor of the Riemann space-time $g_{\mu \nu}(x)$.

The Post-Newtonian approximation is used in relatively weak gravitational fields and in processes where the speeds are small compared with $c$, the speed of propagation of plane wave solutions of the gravitational field in vacuum. For these reasons, Post-Newtonian formulation is enough to describe phenomena within our solar system and the experimental tests performed in it.

The parameter needed to build perturbation series is the quantity $\epsilon=v / c$, and since we will consider $c=1$ for simplicity in the calculations (at last, we will reintroduce the factors with this quantity), we can see that the speeds of the bodies in our solar system $v$ are no greater than $\epsilon$. This means that temporal and spatial derivatives are related to the next relationship:

$\partial_{t} \sim \epsilon \partial_{i}$

where $i=1,2,3$. This relationship implies that all temporal variations are associated with the motion of matter.

As a first step, we will expand the metric tensor $g^{\mu \nu}$,

$g^{00}=1+\stackrel{(2)}{ }^{00}+\stackrel{(4)}{00}^{00}+\cdots$,
$g^{0 j}=\stackrel{(3)}{0 j}^{0 j}+{\stackrel{(5)}{g^{0 j}}+\cdots,}^{\stackrel{(2)}{(4)}}$
$g^{i j}=\eta^{i j}+g^{i j}+g^{i j}+\cdots$.

where $\eta^{i j}$ is the spatial part of the Minkowski metric $\eta^{\mu \nu}$, and $g^{\mu \nu}$ with $l=2,3,4, \ldots$ are terms of the order $\epsilon^{l}$. If we consider the transformation $t \rightarrow-t$, the sign of $\epsilon$ must be changed, too, and for this reason Eqs. (11) and (13) contain only even powers of $\epsilon$, and (12) only odd powers. Eq. (12) 
does not contain $g^{0 j}$ because in the Newtonian approximation $g^{0 j}$ cannot be lower than the second order in $\epsilon$.

We can use Eqs. (11)-(13) to calculate $g_{\mu \nu}$ and its determinant $g=\operatorname{det} g_{\mu \nu}$

$$
\begin{aligned}
& g_{00}=1+\stackrel{(2)}{g_{00}}+\stackrel{(4)}{g_{00}}+\cdots, \\
& g_{0 j}=\stackrel{(3)}{g_{0 j}}+\stackrel{(5)}{g_{0 j}}+\cdots, \\
& g_{i j}=\eta_{i j}+\stackrel{(2)}{g_{i j}}+\stackrel{(4)}{g_{i j}}+\cdots, \\
& g=-1-\stackrel{(2)}{g_{00}}+\stackrel{(2)}{g_{11}}+\stackrel{(2)}{g_{22}}+\stackrel{(2)}{g_{33}}-\stackrel{(4)}{g_{00}}+\stackrel{(4)}{g_{11}}+\stackrel{(4)}{g_{22}}+\stackrel{(4)}{g_{33}} \\
& +g_{00}^{(2)}\left(g_{11}^{(2)}+g_{22}^{(2)}+g_{33}^{(2)}\right)-g_{11}^{(2)} g_{22}^{(2)}-g_{11}^{(2)}(2) \\
& -\stackrel{(2)}{g_{22}} g_{33}^{(2)}+\stackrel{(2)}{g_{12}^{2}}+\stackrel{(2)}{g_{13}^{2}}+\stackrel{(2)}{g_{23}^{2}}+\cdots .
\end{aligned}
$$

If we introduce the quantities

$$
\stackrel{(2)}{z}=\stackrel{(2)}{g_{00}}-\stackrel{(2)}{g} 11-\stackrel{(2)}{g}{ }_{22}-\stackrel{(2)}{g_{33}}
$$

and

$$
\begin{aligned}
& \stackrel{(4)}{z}=\stackrel{(4)}{g_{00}}-\stackrel{(4)}{g}_{11}-\stackrel{(4)}{g_{22}}-\stackrel{(4)}{g}_{33}-\stackrel{(2)}{g_{00}}\left(\stackrel{(2)}{g_{11}}+\stackrel{(2)}{g_{22}}+\stackrel{(2)}{g_{33}}\right)
\end{aligned}
$$

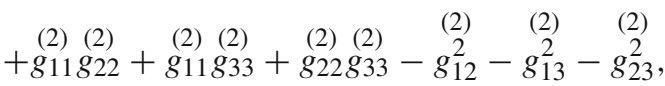

then $g=\operatorname{det} g_{\mu \nu}$ is

$g=-1-\stackrel{(2)}{z}-\stackrel{(4)}{z}$.

Equation (9) in Galilean coordinates are

$\frac{1}{2} g_{00,0}^{(2)}-\frac{1}{2} \eta^{i j} g_{i j, 0}^{(2)}=-\eta^{i j} g_{0 j, i}^{(3)}$,
$\frac{1}{2} g_{00, i}^{(2)}+\frac{1}{2} \eta^{k l} g_{k l, i}^{(2)}=\eta^{k l} g_{i k, l}^{(3)}$,

$$
\begin{aligned}
& {\left[\stackrel{(2)}{g_{00}^{2}}-\stackrel{(4)}{g_{00}}-\frac{1}{2} g_{00}^{(2)} z\right.} \\
& z
\end{aligned}
$$

The expansion of the second rank Ricci tensor written in Cartesian coordinates is

$$
\begin{aligned}
& R_{00}=-\frac{1}{2} \eta^{i j}{\stackrel{(2)}{g_{00}, i j}}_{-}-\frac{1}{2} \eta^{i j}{ }_{g_{00}, i j}^{(4)}-\frac{1}{2}{\stackrel{(2)}{g_{00}, 00}}^{(2)} \\
& +\frac{1}{2} \eta^{k i} \eta^{l j} g_{k l}^{(2)} g_{00, i j}^{(2)}+\frac{1}{2} \eta^{i j} g_{00, i}^{(2)} g_{00, j}^{(2)}+\cdots, \\
& R_{0 j}=-\frac{1}{2} \eta^{i k} g_{0 j, i k}^{(3)}+\cdots, \\
& R_{i j}=-\frac{1}{2} \eta^{k l} g_{i j, k l}^{(2)}+\cdots,
\end{aligned}
$$

where we have used the harmonic gauge conditions (9) to ignore the next terms of Eqs. (24) and (25).

Also, we will expand the energy-momentum tensor of matter, considering it as a perfect fluid, namely,

$T^{\mu \nu}=[p+\rho(1+\Pi)] v^{v} v^{\mu}-p g^{\mu v}$,

where $p$ is the specific isotropic pressure, $\rho$ is the ideal liquid's density, $\Pi$ is the specific self energy, also called internal energy per particle and is the general name for all energies with the except for the rest mass, and $v^{\mu}$ is the four-velocity. The definition of $\Pi$ is given in A first course of general relativity by B. Schutz in [23]

$\Pi=\frac{\rho}{n}-m, \Rightarrow \rho=n(m+\Pi)$,

where $n$ is the number density, the number of particles per unit volume in a momentarily co-moving reference frame.

Tensor (26) must satisfy covariant conservation law given by

$\nabla_{\mu} T^{\mu \nu}=\partial_{\mu} T^{\mu \nu}+\Gamma_{\mu \lambda}^{\mu} T^{\lambda v}+\Gamma_{\mu \lambda}^{v} T^{\lambda \mu}=0$.

The density of the perfect fluid is invariant and must satisfy the covariant continuity equation, that is,

$\frac{1}{\sqrt{-g}}\left(\sqrt{-g} \rho v^{v}\right)_{, v}=0$.

We will expand $T^{\mu \nu}$ in the small parameter $\epsilon$

$$
\begin{aligned}
& T^{00}=\stackrel{(0)}{ }=T^{00}+{ }^{(2)}+T^{00}+T^{(4)}+\cdots, \\
& { }^{(3)} \stackrel{(5)}{0 j}=T^{0 j}+T^{0 j}+\cdots, \\
& T^{i j}+\stackrel{(4)}{i j}=\eta^{i j}+T^{i j}+T^{i j}+\cdots,
\end{aligned}
$$

If gravitational forces are ignored, that is, in Newtonian approximation, the components temporal and spacial of $v^{\mu}$ are

$v^{0}=1+O\left(\epsilon^{2}\right)$ and $v^{i}=v^{i}\left(1+O\left(\epsilon^{2}\right)\right)$.

If we substitute Eqs. (29)-(32) in Eq. (26), we find

$$
\begin{gathered}
{ }^{(0)} \\
T^{00}=\rho, \\
{ }^{(1)} \\
T^{0 j}=\rho v^{j}, \\
{ }^{(0)} \\
T^{i j}=0 .
\end{gathered}
$$

The expanded Einstein field equations (6) are

$$
\begin{aligned}
& \eta^{i j} g_{00, i j}^{(2)}=-8 \pi G T^{00}, \\
& \eta^{i j} g_{00, i j}^{(4)}+g_{00,00}^{(2)}-\eta^{k i} \eta^{l j} \stackrel{(2)}{g_{k l}} g_{00, i j}^{(2)}-\eta^{i j} g_{00, i}^{(2)} g_{00, j}^{(2)} \\
& =-8 \pi G\left(T^{00}+2 g_{00}^{(2)} T^{00}-\eta_{i j} T^{i j}\right),
\end{aligned}
$$




$$
\begin{gathered}
\eta^{j l} g_{0 i, j l}^{(3)}=-16 \pi G \eta_{i j} T^{(1)}, \\
\eta^{k l} g_{i j, k l}^{(2)}=8 \pi G \eta_{i j} T^{(0)} .
\end{gathered}
$$

This set of equations allows us to write the tensor of the Riemann space-time in Newtonian and post-Newtonian approximations.

If we denote $\phi$ as the Newtonian potential, we can suppose that

$\stackrel{(2)}{g}_{00}=-2 \phi$,

then Eq. (36) can be written as

$$
\Delta \phi=-4 \pi G T^{00} .
$$

which is the Poisson equation and $\Delta=\nabla^{2}$ is the Laplacian operator. We consider $\phi$ null at infinity, therefore, our solution is,

$\phi=G \int \frac{T^{00}\left(\mathbf{x}^{\prime}, t\right)}{r} d^{3} x^{\prime}$,

where $r=\left|\mathbf{x}-\mathbf{x}^{\prime}\right|$ is the distance between the source point and observation point (field point).

From Eqs. (38) and (39), we get

$$
\begin{aligned}
& \stackrel{(3)}{g_{0 i}}=-4 G \eta_{i j} \int \frac{T^{0 j}\left(\mathbf{x}^{\prime}, t\right)}{r} d^{3} x^{\prime}, \\
& \stackrel{(2)}{g}_{i j}=2 G \eta_{i j} \int \frac{T^{00}\left(\mathbf{x}^{\prime}, t\right)}{r} d^{3} x^{\prime}=2 \eta_{i j} \phi .
\end{aligned}
$$

If we substitute Eqs. (40), (41) and (44) in Eq. (37), we have $\Delta\left(\stackrel{(4)}{g}{ }_{00}-2 \phi^{2}\right)=-2 \phi, 00+8 \pi G\left(\stackrel{(0)}{T^{00}-\eta_{i j} T^{i j}}\right)$.

(4) must tend to zero at infinity, therefore from Eq. Also, $g_{00}$ me obtain

$$
\begin{aligned}
\stackrel{(4)}{g_{00}=} & 2 \phi^{2}+\frac{1}{2 \pi} \frac{\partial^{2}}{\partial t^{2}} \int \frac{\phi\left(\mathbf{x}^{\prime}, t\right)}{r} d^{3} x^{\prime} \\
& -2 G \int \frac{\left(T^{00}-\eta_{i j} T^{i j}\right)}{r} d^{3} x^{\prime} .
\end{aligned}
$$

Eqs. (19) and (44) can be related by

$\phi_{, 0}=\frac{1}{4} \eta^{i j} g_{0 j, i}^{(3)}$.

Substituting the expanded components of the energy-momentum tensor of matter given by Eqs. (33) and (34) in Eqs. (42) and (43), we get

$\stackrel{(2)}{g_{00}}=-2 \phi, \quad \stackrel{(3)}{g_{0 j}}=4 \eta_{i j} q^{i}, \quad \stackrel{(2)}{g_{i j}}=2 \eta_{i j} \phi$, where

$\phi=G \int \frac{\rho\left(\mathbf{x}^{\prime}, t\right)}{r} d^{3} x^{\prime}$

and

$q^{i}=-G \int \frac{\rho\left(\mathbf{x}^{\prime}, t\right) v^{i}}{r} d^{3} x^{\prime}$.

In the lowest order of approximation, the metric coefficients of the Riemann space-time are

$g_{00}=1-2 \phi, \quad g_{0 j}=4 \eta_{i j} q^{i}, \quad g_{i j}=\eta_{i j}(1+2 \phi)$.

We can use our last results in the covariant conservation law (27) and the covariant continuity equation (28) to find the next approximation for the components of the energy-momentum tensor of matter. We need for this task the Christoffel symbols $\Gamma_{\beta \gamma}^{\alpha}, \sqrt{-g}$ and $u^{0}$ in the zero-order approximation,

$\sqrt{-g}=1+2 \phi$

$v^{0}=1+\phi-\frac{1}{2} v_{j} v^{j}$,

$\Gamma_{00}^{0}=-\phi_{, 0}, \quad \Gamma_{0 i}^{0}=-\phi_{, i}, \quad \Gamma_{00}^{i}=\eta^{i j} \phi_{, j}$,

$\Gamma_{i j}^{0}=-\eta_{i j} \phi_{, 0}+2\left(\eta_{j k} q_{, i}^{k}+\eta_{i k} q_{, j}^{k}\right)$,

$\Gamma_{0 j}^{i}=2 q_{, j}^{i}+\delta_{j}^{i} \phi_{, 0}-2 \eta^{i k} \eta_{j l} q_{, k}^{l}$,

$\Gamma_{j k}^{i}=\delta_{k}^{i} \phi_{, j}+\delta_{j}^{i} \phi_{, k}-\eta^{i l} \eta_{j k} \phi_{, l}$.

Thus, covariant conservation law stays as

$T_{, 0}^{(2)}+T_{, i}^{00}-\rho \phi_{, 0}-2 \rho v^{i} \phi_{, i}=O\left(\epsilon^{5}\right)$,

$T_{, j}^{i j}+\left(\rho v^{i}\right)_{, 0}+\eta^{i j} \rho \phi, j=O\left(\epsilon^{4}\right)$,

the covariant continuity equation will be

$$
\begin{aligned}
& \frac{1}{\sqrt{-g}}\left\{\frac{\partial}{\partial x^{0}}\left(\rho+3 \phi \rho-\frac{1}{2} \rho v_{i} v^{i}\right)+\frac{\partial}{\partial x^{i}}\left(\rho v^{i}+3 \phi \rho v^{i}+\frac{1}{2} \rho v^{i} v^{2}\right)\right\} \\
& =O\left(\epsilon^{4}\right)
\end{aligned}
$$

and the equation of motion of an ideal liquid is given by

$$
\begin{aligned}
\hat{\rho}\left(u_{, 0}^{i}+v^{j} v_{, j}^{i}\right) & =\eta^{i j}\left(-\hat{\rho} \phi, j+p_{, j}\right), \\
\hat{\rho}\left(\Pi_{, 0}+v^{j} \Pi_{, j}\right) & =-p v_{, i}^{i},
\end{aligned}
$$

where $\hat{\rho}=\sqrt{-g} \rho u^{0}$, which is the conserved mass density, which can be written as follows in our approximation

$\hat{\rho}=\rho\left(1+3 \phi-\frac{1}{2} v_{i} v^{i}\right)$,

therefore we can replace $\hat{\rho}$ by $\rho$ which is invariant density.

So, we have the solutions for energy-momentum tensor of matter in the required expansion expressed as

$\stackrel{(2)}{T^{00}}=\rho\left(2 \phi+\Pi-v_{i} v^{i}\right)$, 
(3)

$$
\begin{aligned}
T^{0 j} & =\rho v^{j}\left(2 \phi+\Pi-v_{i} v^{i}\right)+p v^{j}, \\
{ }^{(2)} & \\
T^{i j} & =\rho v^{i} v^{j}-\eta^{i j} p .
\end{aligned}
$$

Now, we can obtain $\stackrel{(4)}{g_{00}}$ given by Eq. (46)

$$
\begin{aligned}
g_{00}^{(4)}= & 2 \phi^{2}+\frac{1}{2 \pi} \frac{\partial^{2}}{\partial t^{2}} \int \frac{\phi\left(\mathbf{x}^{\prime}, t\right)}{r} d^{3} x^{\prime} \\
& -2 G \int \frac{\rho\left(2 \phi+\Pi-2 v_{i} v^{i}\right)+3 p}{r} d^{3} x^{\prime} \\
= & 2 \phi^{2}+\frac{1}{2 \pi} \frac{\partial^{2}}{\partial t^{2}} \int \frac{\phi\left(\mathbf{x}^{\prime}, t\right)}{r} d^{3} x^{\prime} \\
& -4 G \int \frac{\rho \phi}{r} d^{3} x^{\prime}-2 G \int \frac{\rho \Pi}{r} d^{3} x^{\prime} \\
& +4 G \int \frac{\rho v_{i} v^{i}}{r} d^{3} x^{\prime}-6 G \int \frac{p}{r} d^{3} x^{\prime} \\
= & 2 \phi^{2}+\frac{1}{2 \pi} \frac{\partial^{2}}{\partial t^{2}} \int \frac{\phi\left(\mathbf{x}^{\prime}, t\right)}{r} d^{3} x^{\prime} \\
& -4 \Phi_{1}-2 \Phi_{2}-4 \Phi_{3}-6 \Phi_{4},
\end{aligned}
$$

where

$\Phi_{1}=G \int \frac{\rho \phi}{r} d^{3} x^{\prime}, \Phi_{2}=G \int \frac{\rho \Pi}{r} d^{3} x^{\prime}$,

$\Phi_{3}=-G \int \frac{\rho v_{i} v^{i}}{r} d^{3} x^{\prime}, \Phi_{4}=G \int \frac{p}{r} d^{3} x^{\prime}$,

$\Phi_{1}, \Phi_{2}, \Phi_{3}$ and $\Phi_{4}$ are the generalized gravitational potentials. Now we have obtained the metric coefficients of $g_{\mu \nu}$ of the metric of the Riemann space-time, that is

$$
\begin{aligned}
g_{00}= & 1-\frac{2 \phi}{c^{2}}+\frac{1}{c^{4}}\left\{\phi^{2}-G \frac{\partial^{2}}{\partial t^{2}} \int \rho\left(\mathbf{x}^{\prime}, t\right) r d^{3} x^{\prime}-4 \Phi_{1}\right. \\
& \left.-2 \Phi_{2}-4 \Phi_{3}-6 \Phi_{4}\right\}+O\left(\epsilon^{6}\right) \\
g_{0 j}= & \frac{4}{c^{3}} \eta_{i j} q^{i}+O\left(\epsilon^{5}\right) \\
g_{i j}= & \frac{1}{c^{2}} \eta_{i j}(1+2 \phi)+O\left(\epsilon^{4}\right)
\end{aligned}
$$

where we have explicitly written the dependence of inverse factors on $c$, and we have used the identity

$\frac{1}{2 \pi} \int \frac{\phi}{r} d^{3} x^{\prime}=-G \int \rho\left(\mathbf{x}^{\prime}, t\right) r d^{3} x^{\prime}$

demonstrated in [24].

We can write our system of Eqs. (36)-(39) as follows

$\Delta \phi=-4 \pi G \rho$,

$\Delta \mathbf{q}=-4 \pi G \rho \mathbf{v}$,

$\Delta \Psi=-2 \frac{\partial^{2} \phi}{\partial t^{2}}+8 \pi G \rho\left(2 \phi+\Pi+2 v^{2}+3 \frac{p}{\rho}\right)$,

where $\Psi=\psi-2 \phi^{2}$ and $\psi=\stackrel{(4)}{g_{00}}$.

$\eta_{i j} \Delta \phi=-4 \eta_{i j} \pi G \rho$
Condition (47) is written as

$\frac{\partial \phi}{\partial t}+3 \mathbf{q}=0$.

From the system (65)-(69), we have a solution for $\phi$, the ordinary Newtonian scalar potential given by Eq. (49)

$\phi(\mathbf{x}, t)=G \int \frac{\rho\left(\mathbf{x}^{\prime}, t\right)}{r} d^{3} x^{\prime}$.

The Heaviside vector potential whose components are given by Eq. (50) is written in vector form as follows

$\mathbf{q}(\mathbf{x}, t)=-G \int \frac{\rho\left(\mathbf{x}^{\prime}, t\right) \mathbf{v}}{r} d^{3} x^{\prime}$.

We define the potentials $\Xi$ and $\chi$ to write a simplified solution to Eq. (67)

$\Xi=G \int \frac{\rho\left(\mathbf{x}^{\prime}, t\right)\left(2 \phi+\Pi+2 v^{2}+\frac{3 p}{\rho}\right)}{r} d^{3} x^{\prime}$,

and

$\chi=G \int \rho\left(\mathbf{x}^{\prime}, t\right) r d^{3} x^{\prime}$,

therefore the solution to Eq. (67) is

$\Psi=-2 \Xi-\frac{\partial^{2} \chi}{\partial t^{2}}$.

We define the total contribution of the scalar and vector potentials $\phi_{t}$ and $\mathbf{q}_{t}$ as

$\phi_{t}=-\frac{1}{2}\left(-1+g_{00}\right)=\stackrel{(0)}{\phi}+\left(\begin{array}{l}(2) \\ \phi^{2}-\stackrel{(2)}{\Theta}\end{array}\right)+O\left(\epsilon^{4}\right)$,

where, for convenience, we have introduced $\Theta=\frac{1}{2} \Psi$ and

$\mathbf{q}_{t}=\frac{1}{4} \stackrel{(2)}{g}_{0 j}+O\left(\epsilon^{4}\right)=\stackrel{(2)}{q}_{j}+O\left(\epsilon^{4}\right)$.

In both equations, we have reduced the order of the potentials because we have multiplied Eq. (74) by $c^{2}$ and Eq. (75) by $c$ in the given definitions. Then, we can define the ordinary gravitational vector $\mathbf{g}$ and the Heaviside field vector $\mathbf{k}$ as functions of the scalar and vector potentials $\phi_{t}$ and $\mathbf{q}_{t}$

$\mathbf{g}=-\nabla \phi_{t}-\frac{\partial \mathbf{q}_{t}}{\partial t}=-\nabla^{\stackrel{(0)}{\phi}}+\nabla \stackrel{(2)}{\Theta}-\nabla\left(\stackrel{(2)}{\phi^{2}}\right)-\frac{1}{3} \frac{\partial^{2}}{\partial t^{2}} \nabla^{(2)}$

and

$\mathbf{k}=\nabla \times \mathbf{q}_{t}$.

So, in this way, we can obtain the second-order Maxwelllike formulation of the gravitational field equations, once we have reintroduced the factors $c^{-2}$,

$$
\nabla \cdot \mathbf{g}=-4 \pi G \rho\left\{1-\frac{2}{c^{2}}\left(2 \phi+\Pi+2 v^{2}+3 \frac{p}{\rho}\right)\right\}
$$




$$
\begin{gathered}
-\frac{4 \pi G}{3 c^{2}} \frac{\partial^{2} \rho}{\partial t^{2}}+O\left(\epsilon^{4}\right), \\
\nabla \cdot \mathbf{k}=O\left(\epsilon^{4}\right), \\
\nabla \times \mathbf{g}=-\frac{\partial \mathbf{k}}{\partial t}, \\
\nabla \times \mathbf{k}=-\frac{4 \pi G}{c^{2}} \mathbf{J}+\frac{1}{c^{2}} \frac{\partial \mathbf{g}}{\partial t}+O\left(\epsilon^{4}\right) .
\end{gathered}
$$

System of Eqs. (78)-(81) are our second order Jefimenko equations for gravitation. We should note that although the potential Heaviside vector in Eq. (75) is of the order $\epsilon^{2}$, this factor disappears in Eq. (80) because when we calculate the curl of $\mathbf{g}$ and the time derivative of $\mathbf{k}$ and equalise both amounts, after using vector identities from [10], we find that Eq. (80) does not contain terms of orders greater or equal than $\epsilon^{2}$.

The analogous of the Lorentz force is given if we use $\kappa=1+2 \phi$, the coefficient of the spatial part of the postNewtonian metric and $v^{0}$ from Eq. (53) in the geodesic equation,

$$
\frac{d}{d t}\left(\kappa v^{0} \mathbf{v}\right)=v^{0}\left(\mathbf{g}+\mathbf{v} \times \mathbf{k}+v^{2} \nabla \kappa\right)+O\left(\epsilon^{4}\right),
$$

after substituting $\kappa$ and $u^{0}$ we have

$$
\begin{aligned}
& \frac{d}{d t}\left(1+3 \phi-\frac{1}{2} v^{2}\right) \mathbf{v} \\
& =\left(1+\phi-\frac{1}{2} v^{2}\right)(\mathbf{g}+\mathbf{v} \times \mathbf{k})+O\left(\epsilon^{4}\right) .
\end{aligned}
$$

Also, we can establish the zero-order of approximation, which gives us the Newtonian theory, formulated as a force field theory in the following way

$\nabla \cdot \mathbf{g}=-4 \pi G \rho$

and

$\nabla \times \mathbf{g}=0$.

Equation (84) leads us to the Laplace equation for the scalar potential $\phi$ and Eq. (85) indicates us that the ordinary gravitational field is conservative.

\section{Conclusions}

Jefimenko obtained his analogous of the Maxwell equations for gravitation, by postulating an analogy between his retarded solutions for electromagnetic field and retarded solutions for the gravitational field, and assuming the existence of a second field analogous to the magnetic field in electrodynamics. This set of solutions given in $[9,10]$ led to Jefimenko equations analogous to Maxwell equations (1)(4).

From the set of second-order Jefimenko equations (78), (79), we can establish that the system obtained by Jefimenko is correct because the limit in the first order of our non-linear gravitational equations gives us the system obtained originally by Jefimenko for the fields $\mathbf{g}$ and $\mathbf{k}$. The idea of Jefimenko related to establishing the electromagnetic analogy of the gravitational field is true, and it was worked by various authors [19,20] and by ourselves, in [25-27], but we want to cite again the first time when this analogy was postulated, we refer to the work of Oliver Heaviside published more than a century ago in [1], work eclipsed by the appearance of the general relativity theory in 1915 in his paper titled Feldgleichungen der Gravitation [5].

The set of second-order gravitational equations (78), (79) can be obtained also from the Logunov's equations gotten in the relativistic theory of gravitation, but in this theory gravitation is considered as a tensor field in the Minkowski space-time, which means that Logunov considered the metric of the Riemann space-time as the contribution of a gravitational tensor field with a Minkowski background. Whereas in general relativity the metric of the Riemann space-time for weak gravitational fields is considered as the sum of the Minkowski metric plus a perturbation. In Logunov's theory is considered the effective Riemann space-time as the contribution of the gravitational field tensor $\Phi^{\mu v}$ which depends on the coordinates of Minkowski space-time, whereas in general relativity the metric tensor of Riemann space-time $g_{\mu \nu}$ is the field itself. Both theories give us the same predictions for weak gravitational fields, which means that using PostNewtonian approximation we obtain the same experimental results using the first or the last. We have already seen that contrary to the general belief Einstein saw as the most important achievement of his general relativity theory the unification of gravity and inertia instead of geometrizing gravity. On the other hand, Logunov's theory makes a clear distinction between gravity and inertia.

We have found that the limit of the non-linear expressions (78)-(81) called second-order Jefimenko equations are the Jefimenko equations given by (1)-(4) as a first order of approximation. Whereas in regions where matter can be considered at rest (or in uniform movement) in the respective inertial reference system, this system leads us to the zeroorder of approximation, called Newtonian theory and given by Eqs. (84), (85).

Acknowledgements We would like to thanks all people working in CONACyT, for the economical support given to the doctoral student M. D. David A. Pérez Carlos. Also, M. D. David A. Pérez Carlos wants to give acknowledgement to Olga Aleksandrovna Veselkova for her unconditional support, for the advice she has given him to be better academically and as a person.

Data Availability Statement This manuscript has no associated data or the data will not be deposited. [Authors' comment: Data sharing not applicable to this article as no datasets were generated or analysed during the current study.] 
Open Access This article is licensed under a Creative Commons Attribution 4.0 International License, which permits use, sharing, adaptation, distribution and reproduction in any medium or format, as long as you give appropriate credit to the original author(s) and the source, provide a link to the Creative Commons licence, and indicate if changes were made. The images or other third party material in this article are included in the article's Creative Commons licence, unless indicated otherwise in a credit line to the material. If material is not included in the article's Creative Commons licence and your intended use is not permitted by statutory regulation or exceeds the permitted use, you will need to obtain permission directly from the copyright holder. To view a copy of this licence, visit http://creativecomm ons.org/licenses/by/4.0/.

Funded by $\mathrm{SCOAP}^{3}$.

\section{References}

1. O. Heaviside, Electr. 31, 5125-5134 (1893)

2. W. Pauli, Theory of relativity (Dover publications Inc, New York, 1981)

3. S. Weinberg, Gravitation and cosmology: principles and applications of the general theory of relativity (Wiley and sons, Inc., London, 1972)

4. C.W. Misner, K.S. Thorn, J.A. Wheeler, Gravitation (Princeton University Press, Princeton, 2017)

5. A. Einstein, Sitzungsber. Preuss. Akad. Wiss. Berlin (Math. Phys.), (1915)

6. A.A. Logunov, M.A. Mestvirishvili, Found. Phys. 16, 1 (1986)

7. A.A. Logunov, YuM Loskutov, M.A. Mestvirishvili, Prog. Theor. Phys. 80, 6 (1988)

8. A.A. Logunov, M.A. Mestvirishvili, The relativistic theory of gravitation (Mir publishers, Moscow, 1989)

9. O. Jefimenko, Causality, electromagnetic induction and gravitation: A different approach to the theory of electromagnetic and gravitational fields, 2nd edn. (Princeton University Press, Princeton, 2000)

10. O. Jefimenko, Gravitation and Cogravitation: Developing Newton's Theory of Gravitation to its Physical and Mathematical Conclusion, 1st edn. (Electret Scientific Star City, Waynesburg, 2006)

11. B. Vetô, Eur. J. Phys. 31(5), 1123 (2010)

12. B. Vetô, Eur. J. Phys. 32(5), 11323 (2011)

13. I. Ciufolini, E.C. Pavlis, Nature 431(7011), 958-960 (2004)

14. L. Iorio, Celest. Mech. Dyn. Astron. 79(3), 201-230 (2001)

15. J. A. Wheeler Geons, black holes, and quantum foam: a life in physics, (Northon and Company, 2000)

16. R.P. Feynman, F. Moringo, $W$ (Addison-Wesley Publishing Company, London, G. Wagner. Feynman lectures on gravitation, 1995)

17. D. Lehmkuhl, Stud. Hist. Philos. Mod. Phys. 46, 316-326 (2014)

18. A. Einstein, Vierteljahrschirift für gerichtliche medizin 44, 37-40 (1912)

19. A.I. Arbab, Astrophys. Space Sci. 330, 61-68 (2010)

20. W. S. González, Gravitoelectromagnetismo y principio de Mach (eWT Ediciones, Cádiz, 2013)

21. E.B. Fomalont, S.M. Kopeikin, Astrophys. J. 598(1), 704-711 (2003)

22. V.A. Fock, The theory of space, time and gravitation, 2nd edn. (Pergamon press LTD, Oxford, 1964)

23. B. Schutz, A first course in general relativity (Cambridge University Press, Cambridge, 2009)

24. V.S. Vladimirov, Equations of mathematical physics (MIR publishers, Moscow, 1984)

25. D. Pérez Carlos, Augusto Espinoza, Andrew Chubykalo, O. A. J. Phys. 2 (1),1-12, (2018)

26. A. Espinoza, A Chubykalo, D. Pérez Carlos, J.Mod.Phys.,7, 16171626, (2016)

27. A. Chubykalo, A. Espinoza, D. Pérez Carlos, Conceptual Content of the Generalized Theory of Gravitation of Jefimenko. J. Mod. Phys., 9, 1522-1544, (2018) 